Article

\title{
National and Sub-National Social Distancing Responses to COVID-19
}

\author{
Terrence Iverson * (D) and Edward Barbier (D)
}

check for updates

Citation: Iverson, Terrence, and Edward Barbier. 2021. National and Sub-National Social Distancing Responses to COVID-19. Economies 9: 69. https://doi.org/10.3390/ economies 9020069

Academic Editor: Sergio Scicchitano

Received: 13 January 2021

Accepted: 16 April 2021

Published: 1 May 2021

Publisher's Note: MDPI stays neutral with regard to jurisdictional claims in published maps and institutional affiliations.

Copyright: (C) 2021 by the authors. Licensee MDPI, Basel, Switzerland. This article is an open access article distributed under the terms and conditions of the Creative Commons Attribution (CC BY) license (https:/ / creativecommons.org/licenses/by/ $4.0 /)$.

\begin{abstract}
Department of Economics, Colorado State University, Fort Collins, CO 80523, USA; edward.barbier@colostate.edu * Correspondence: terry.iverson@colostate.edu
\end{abstract}

\begin{abstract}
We examine the effectiveness of sub-national actions to control a novel disease, such as COVID-19, in the absence of national policy. Evidence shows that countries where sub-national governments have undertaken unilateral social distancing measures to combat the pandemic with little or no coordination have performed less well in controlling the spread of the disease. We explore analytically whether agreement on a common social distancing policy among sub-national governments, i.e., states or provinces, can lead to a better outcome than if each state or province pursues its own social distancing policy in isolation. A key feature of our model is that it accounts for the inter-jurisdictional spillover effects of each sub-national jurisdiction's policy choice with respect to social distancing. Our results show that, in the absence of a national mandatory agreement, a sub-national agreement with sufficient coordination of social distancing policy among states yields a more effective and efficient control of a pandemic compared to states choosing policy unilaterally. These findings strongly support calls for greater cooperation among and assistance for sub-national governments to improve the effectiveness of their social distancing efforts in controlling the pandemic.
\end{abstract}

Keywords: COVID-19; national policy; social distancing; sub-national jurisdiction

\section{Introduction}

In response to the COVID-19 pandemic, national and sub-national governments have adopted a wide range of policies and targeted actions to cope with the public health emergency and its economic impact (Hale et al. 2020a). Among these, the dominant public health strategy has been social distancing (Cheng et al. 2020; GMF Experts 2020; Imai et al. 2020; Nouvellet et al. 2020). Such actions include physical distancing rules, stay-at-home orders, school closures, lockdowns, curfews, closing of non-essential businesses, banning of mass gatherings, quarantining and similar regulations to restrict person-to-person contact (Cheng et al. 2020). Preliminary evidence suggests that social distancing actions have been effective in controlling the spread of COVID-19 (Chu et al. 2020; Imai et al. 2020; Nouvellet et al. 2020).

When designing and implementing social distancing policies within countries, a key consideration is the presence of inter-jurisdictional spillovers. In the spring and summer of 2020, such spillovers were prominent among the various states in the US. For example, the spring lockdown in New York and other northeastern states benefited residents in Florida (and elsewhere) who did not adopt such stringent social distancing policies to control the coronavirus locally. However, the continuing absence of a lockdown in Florida well into summer 2020 likely lead to health and economic costs in New York and other states in the fall.

Such inter-jurisdictional spillovers suggest a strong case for setting social distancing policies at a national scale. Unfortunately, most public health actions to date have been instigated or enforced by sub-national governments. One reason is that sub-national governments in most countries are responsible for the provision of essential public goods such as health, public order and safety or social protection, which are essential for combatting pandemics. Another reason is that COVID-19 impacts have varied significantly 
across regions within countries, so sub-national governments have often needed to interpret, modify and adapt national social distancing guidelines and policies to suit local and regional conditions (Cheng et al. 2020; Organization for Economic Cooperation and Development OECD, OECD). The absence of binding national policies raises important policy questions. Can sub-national actions be an effective replacement in controlling the outbreak of a disease? To what extent can policy coordination of social distancing among sub-national jurisdictions approximate the outcome of an effective national policy? How does the effectiveness of sub-national action change when some states or provinces refuse to participate?

To our knowledge, this is the first paper to explore analytically whether agreement on a common social distancing policy among sub-national governments, i.e., states or provinces, can lead to a more effective national outcome than if each state or province pursues its own policy in isolation. A key feature of our model is that it accounts for the inter-jurisdictional spillover effects of each sub-national jurisdiction's choice of social distancing policy. Each sub-national government (henceforth a "state") chooses its own level of social distancing, but the aggregate level of infection in the country is determined by the combined level of social distancing across all states. Local deaths in turn are determined by aggregate infection and local policy.

We explore three institutional settings that determine social distancing for each state: a mandatory national policy that all states must adopt, a laissez-faire setting where each state sets its own policy, and a sub-national agreement where states form a coalition and choose policy collectively. Under both the laissez-faire setting and the sub-national agreement, we also examine what happens when some states opt out, choosing to keep their economy open rather than impose strict social distancing. All outcomes are compared to the benchmark of a mandatory national policy.

Our analysis shows that a national policy entails higher social distancing and lower aggregate infection compared to the laissez-faire case. Under laissez faire, states do not take into account the impact of their choice on other states; thus, the chosen policy is less stringent than would be collectively optimal. When some states opt out of social distancing, the other states take this into account and end up social distancing more. If the fraction of states without a policy is sufficiently high, the optimal response of the remaining states entails more social distancing than under national policy. Despite this, aggregate infection is higher, so participating states are worse off both in terms of disease burden and economic costs.

Next, we consider a sub-national agreement in which a subset of states cooperate, jointly pursuing social distancing policy that is optimal for the group. When all states participate, the outcome coincides with the national agreement. However, when some states opt out, the participating states always distance more, though aggregate infection remains higher. While the sub-national agreement cannot make up for the absence of a national agreement when there are non-participants, it still states yields a more effective and efficient control of the pandemic compared to states choosing policy unilaterally without coordination.

\section{Background}

The CoronaNet Research Project has compiled a global database on government responses to the coronavirus based on nearly 13,890 announced policies across more than 190 countries (Cheng et al. 2020). Of these policies, 6778 (48.8\%) can be categorized as social distancing actions, such as closure and regulation of schools, curfews, internal border restrictions, lockdowns, quarantines, restriction and regulation of businesses, restriction and regulation of government services, restrictions of mass gatherings and "social distancing" rules-i.e., policies that limit physical contact between individuals to at least $1.5 \mathrm{~m}$ or 6 feet. Provincial, state and municipal governments were responsible for instigating 47.2\% of these social distancing policies and enforcing $78.5 \%$ of them. As far as implementing 
social distancing, "subnational governments are at the frontline of the COVID-19 response" (Organization for Economic Cooperation and Development OECD, p. 6).

However, a consequence of this de-centralized approach to social distancing is that it may be less effective in controlling the spread of the virus through a country's population. Health outcomes enacted at the state or local level, such as control of the COVID-19 disease outbreak through social distancing, are local public goods with strong inter-jurisdictional spillovers (GMF Experts 2020; Pineda and Radics 2020; Ponce-Rodríguez et al. 2018). An individual infected in a state or province with weak social distancing rules could transmit the disease when traveling to another state or province, even if the latter jurisdiction has a stronger social distancing policy. Equally, if a sub-national government decides to opt out of social distancing, or relax its rules prematurely, any spike in transmission could easily spread to other jurisdictions. The potential spillover dangers in controlling the COVID-19 pandemic from sub-national governments adopting unilateral social distancing policies are summarized by Pineda and Radics (2020): "if each state or municipality were to implement their own, separate isolation rules, the resulting people movement patterns could undermine contagion-reduction efforts. While autonomous decision-making is a basic feature of decentralization, in areas where the general wellbeing is at stake, coordinated action is preferable."

There may be many reasons why sub-national governments have chosen unilateral actions over coordination in response to the pandemic. In the United States, with the absence of a national policy, the failure or slowness of some states to adopt social distancing measures is largely attributed to political differences and divisions between states (Adolph et al. 2020). Although some coordination of social distancing has occurred among a few neighboring states, the hands-off approach of the US federal government in favor of a unilateral approach by states has led to one of the highest deviations in sub-national adoption of social distancing policies globally (GMF Experts 2020; McKenzie and Adams 2020; Organization for Economic Cooperation and Development OECD). Other countries may have adopted a coordinated approach to social distancing among sub-national governments initially, but then differences have led to deviations from that consensus. For example, in Germany, increasing concerns about the social and economic harms done by prolonged social distancing measures led to some states openly disregarding and diverging from agreements with other state governments over a common policy (Büthe et al. 2020). In the United Kingdom, disagreement over the public health implications of relaxing social distancing rules led to Scotland, Wales, Northern Ireland and England independently choosing different policies (BBC 2020).

Evidence is emerging that countries where sub-national governments have undertaken unilateral social distancing measures to combat the pandemic with little or no coordination have performed less well in controlling the spread of the disease (Hale et al. 2020b; Imai et al. 2020; McKenzie and Adams 2020; GMF Experts 2020; Nouvellet et al. 2020). Due to the lead role of sub-national governments in implementing social distancing during the pandemic, this has led to calls for greater policy coordination and agreement among states and provinces to improve their effectiveness in controlling the pandemic (GMF Experts 2020; Organization for Economic Cooperation and Development OECD; Pineda and Radics 2020). Such views are backed by studies showing that control of disease and other health outcomes with local public good characteristics and inter-jurisdictions spillovers are enhanced by sub-national agreements and coordination over policy (Leland et al. 2020; Ponce-Rodríguez et al. 2018; Spicer et al. 2010).

In many countries, state and provincial governments were responsible for instigating and enforcing social distancing measures, and for some countries, there were considerable differences in the stringency of policies adopted by different sub-national jurisdictions. For example, McKenzie and Adams (2020) found that countries with the highest variation in sub-governmental social distancing measures, such as Nigeria, Uruguay, Australia, the United States and Canada, ranked among the lowest stringency of overall social distancing policy at the national level. In contrast, New Zealand and European countries such as 
France and Italy had the least differences in policies adopted at the sub-national level and also ranked high in overall national stringency of social distancing. This raises important questions about the effectiveness of imposing social distancing, especially when there is an ineffective or non-existent policy at the national level, and local jurisdictions vary considerable in the level of social distancing they choose to implement. To explore further these questions, we develop a model of social distancing policy in a country is divided into sub-national jurisdictions.

Most economic models of social distancing policy consider the instance in which policy is implemented at a national scale. Examples include Eichenbaum et al. (2020), Acemoglu et al. (2020), and Peri et al. (2021). In addition, a variety of empirical studies have attempted to quantify the spillovers associated with social distancing policies for neighboring regions. Holtz et al. (2020) use mobile phone and social media data to quantify policy spillovers across state lines. They conclude that uncoordinated state-led policies have substantial social cost relative to a setting with coordinated national policy. Lin and Meissner (2020) also show that interstate spillovers are important. Using a regression discontinuity design with county-level data, they show that both the spread of COVID cases and the economic cost of policy are impacted by social distancing policies in neighboring states.

Our study of the economic inefficiency that arises when independent jurisdictions regulate a problem with transboundary spillovers is a general problem type that has been widely studied in economics. For example, the problem of regulating a transboundary pollutant by sovereign nation states is a major topic in Trade Theory and Environmental Economics (Copeland and Taylor 1995; Sandler 1997; Somanathan et al. 2014). Similarly, the challenging of regulating spillovers from a transboundary epidemic has also been widely recognized. Nevertheless, while the basic intuition for the inefficiency is the same in our case, we have not seen any attempts to theoretically model the inefficiency that arises when policy is implemented by sub-national jurisdictions, along the potential to reduce this inefficiency through sub-national cooperation that internalizes the collective spillover. This topic is, to our knowledge, unstudied.

\section{Model: Social Distancing with Sub-National Jurisdictions}

The model assumes a large fixed population in a country that is initially free from a new contagious virus (e.g., COVID-19). The standard assumption in basic disease models is that a novel virus like COVID-19 will spread through the population based on contact and transmission between infected and susceptible individuals, and an epidemic will occur depending on whether or not the number of infected individuals initially increases or falls (Cobey 2020; Diekmann and Heesterbeek 2000; Eubank et al. 2020; Hethcote 2009; van den Driessche 2017). If an epidemic occurs, this initial, or basic, reproduction rate of the virus, usually denoted $R_{0}$, also determines the final size of the epidemic in terms of the number of infected individuals and deaths. Absent a vaccine, the primary public health policy for controlling the initial outbreak of an epidemic is to reduce $R_{0}$ through social distancing measures that reduce contact and transmission (Cobey 2020; Eubank et al. 2020; Ferguson et al. 2020).

To study the problem of optimal policy when there are cross-jurisdictional spillovers, we develop a simplified static model that resembles the essential long run features of the dynamic contagion models used in the epidemiological literature. In Appendix A, we develop a single-region dynamic model to justify the static model developed here. We show that social distancing not only reduces $R_{0}$, but also the final number of infected and the cumulative number of deaths at the end of the outbreak. If $R_{0}>1$, the number of infected individuals initially grows exponentially (see Equation (A1)); while if $R_{0}<1$, the disease dies out. The total number of susceptible individuals who succumb to the virus and possibly die in the course of the epidemic is also determined by $R_{0}$. Consequently, if $R_{0}$ is large, the final size of the population infected during the epidemic will be significant and the number of expected deaths will also be high (Equation (A4)). The derivation in 
Appendix A justifies our assumption of a monotonic relationship between the constant level of social distancing chosen in an individual jurisdiction and the long run rate of infection.

To model cross-jurisdictional spillovers, we assume there is a unit continuum of identical jurisdictions (or "states") in a country that faces an outbreak of COVID-19. Each state $i$ chooses a level of social distancing, $d_{i}$, that reduces the time its citizens spend interacting outside the home. To model the endogenous level of aggregate infection and local deaths, we adopt two further assumptions. First, we assume that aggregate infection in the country, $I$, is a linear function of local policy in all states:

$$
I=\alpha \int_{0}^{1}\left(1-d_{j}\right) d j
$$

The adopted functional form assumes linear mixing between spatial regions, an assumption discussed, for example, in (Ball et al. 2011). The parameter $\alpha>0$ depends both on the basic reproductive number $R_{0}$ and the rate of social mixing across jurisdictions.

Second, as in Atkeson (2020), we assume that deaths are a multiplicative function of net social distancing and aggregate infection. Specifically, deaths $D_{i}$ in state $i$ depend on local distancing policy and on the aggregate level of infection that obtains nationally:

$$
D_{i}=\left(1-d_{i}\right) \gamma I=\left(1-d_{i}\right) \alpha \gamma \int_{0}^{1}\left(1-d_{j}\right) d j, \gamma>0 .
$$

In all states, the value of a statistical live is $V>0$, while the local cost of distancing is $c d_{i}$ for $c>0$. To ensure policy is sufficiently valuable to make the problem interesting, we assume $\alpha \gamma V>c$. It follows that the objective of each jurisdiction is to minimize the sum of mortality costs and social distancing costs:

$$
V D_{i}+c d_{i}
$$

\section{Policy Equilibria}

The determination of social distancing policy depends on the institutional setting. We consider three alternatives. The algebraic solutions for each case are presented in Sections 3 and 4 . Section 5 presents and discusses a graphical summary of the main results.

\subsection{National Policy}

Since all states are identical, national policy consists of a mandatory distancing policy that all states must follow. Chosen to minimize total social costs for the representative state, it solves

$$
\min _{0 \leq d^{N} \leq 1}\left\{V\left(1-d^{N}\right) \alpha \gamma \int_{0}^{1}\left(1-d^{N}\right) d j+c d^{N}\right\} .
$$

The assumption $\alpha \gamma V>c$ ensures the solution is interior. Optimal policy is

$$
d^{N}=1-\frac{c}{2 \alpha \gamma V} .
$$

The corresponding level of infection is

$$
I^{N}=\alpha\left(1-d^{N}\right)=\frac{c}{2 \gamma V} .
$$

\subsection{Laissez Faire}

Next, consider the situation without national policy. Since each state has zero mass, it does not internalize the impact of its own policy on the aggregate level of infection. It thus takes $I$ as given and chooses $d_{i}$ to solve

$$
\min _{0 \leq d_{i} \leq 1}\left\{V\left(1-d_{i}\right) \gamma I+c d_{i}\right\}
$$


In equilibrium, aggregate infection is determined by the combined policy of all states, implying a fixed-point condition. We first consider the equilibrium in which all states participate, then we consider the impact on outcomes and equilibrium policy when some states opt out.

\subsubsection{Full Participation}

Because the objective function is linear in the control, state $i$ will choose $d_{i}=0$ if $c>V \gamma I, d_{i}=1$ if $V \gamma I>c$, and $d_{i} \in(0,1)$ if $V \gamma I=c$. Working through each case, the only fixed-point equilibrium occurs when $d_{i}$ is interior. Thus, $V \gamma I=c$.

Since states are identical, we only consider symmetric equilibria. We denote the full-participation state-led policy by $d^{F}$. It solves

$$
V \gamma \alpha\left(1-d^{F}\right)=c .
$$

Thus,

$$
d^{F}=1-\frac{c}{\alpha \gamma V} .
$$

The assumption $\alpha \gamma V>c$ ensures $d_{F} \in(0,1)$.

The corresponding level of infection is

$$
I^{F}=\frac{c}{\gamma V} .
$$

It is straightforward to show that

$$
d^{F}=1-\frac{c}{\alpha \gamma V}<1-\frac{c}{2 \alpha \gamma V}=d^{N} .
$$

while

$$
I^{F}=\frac{c}{\gamma V}>\frac{c}{2 \gamma V}=I^{N}
$$

State-led policy entails lower social distancing and higher aggregate infection. Unlike the national government, states do not internalize the burden that their contribution to aggregate infection imposes on other states. Society is better off if policy is set at a national scale.

\subsubsection{Incomplete Participation}

Next, suppose fraction $\phi$ of states opt out of social distancing measures entirely, choosing $d_{i}=0$. States might do this for various reasons, including political or ideological ones, though the origin of their behavior is outside the model.

The remaining states do what is locally optimal, taking as given the equilibrium level of aggregate infection. The choice problem for participating states remains the same as described in Equation (5) with the difference that the equilibrium level of infection that states correctly anticipate is now affected by the absence of social distancing in nonparticipating states.

Since participating states are identical, we look for a symmetric equilibrium (i.e., where all participating states do the same thing). Let $d^{I}$ denote the incomplete-participation stateled policy. Given a candidate $d^{I}$, aggregate infection is

$$
I=\alpha\left[\phi+(1-\phi)\left(1-d^{I}\right)\right]
$$

As before, the condition for an interior equilibrium is

$$
V \gamma \alpha\left[\phi+(1-\phi)\left(1-d^{I}\right)\right]=c
$$


Solving for $d^{I}(\phi)$ and imposing the upper bound implies

$$
d^{I}=\left\{\begin{array}{l}
\frac{1}{1-\phi}\left[1-\frac{c}{\alpha \gamma V}\right], \text { for } \phi<\frac{c}{\alpha \gamma V} \\
1, \text { for } \phi \geq \frac{c}{\alpha \gamma V}
\end{array}\right.
$$

The equilibrium policy reduces to the full participation case when $\phi=0$. As $\phi$ increases, $d^{I}$ increases until the threshold $\hat{\phi}=\frac{c}{\alpha \gamma V}$, after which $d^{I}=1$. The assumption $\alpha \gamma V>c$ ensures $\hat{\phi}<1$.

The corresponding level of infection is

$$
I^{I}=\left\{\begin{array}{l}
\frac{c}{\gamma V}, \text { for } \phi<\frac{c}{\alpha \gamma V} \\
\phi \alpha, \text { for } \phi \geq \frac{c}{\alpha \gamma V}
\end{array}\right.
$$

\section{Sub-National Agreement}

Finally, suppose the participating states form a coalition, acting together to pick the distancing level that is collectively optimal. A candidate sub-national agreement is a target $\hat{d}$ that all participants implement.

Aggregate infection under the agreement is

$$
I=\alpha[\phi+(1-\phi)(1-\hat{d})] .
$$

The policy that minimizes total social costs for the coalition solves

$$
\min _{0 \leq \hat{d} \leq 1}\{\alpha \gamma V(1-\hat{d})[\phi+(1-\phi)(1-\hat{d})]+c \hat{d}\}
$$

To solve this, define $\theta=1-\hat{d}$ and $\Gamma=\alpha \gamma V$. Then, the problem can be written as

$$
\min _{0 \leq \theta \leq 1} c+[\phi \Gamma-c] \theta+(1-\phi) \Gamma \theta^{2}
$$

If the term in brackets is non-negative, then the solution is a corner solution with $\theta=0$, which implies $\hat{d}=1$. This is true provided $\phi$ is greater than or equal to $\hat{\phi}$ such that $\hat{\phi} \Gamma-c=0$.

Thus, $\hat{\phi}=\frac{c}{\alpha \gamma \nabla}$.

If $\phi<\hat{\phi}$, then the solution is interior and satisfies the first-order condition:

$$
\phi \Gamma-c+2(1-\phi) \Gamma \theta=0
$$

This implies

$$
\theta=\frac{1}{2} \frac{1}{1-\phi}\left[\frac{c}{\alpha \gamma V}-\phi\right]
$$

Thus,

$$
\hat{d}=1-\frac{1}{2} \frac{1}{1-\phi}\left[\frac{c}{\alpha \gamma V}-\phi\right]
$$

It is easy to show that this coincides with the national policy when $\phi=0$.

Combining the results above, the equilibrium sub-national agreement takes the form

$$
\hat{d}=\left\{\begin{array}{l}
1-\frac{1}{2(1-\phi)}\left(\frac{c}{\alpha \gamma V}-\phi\right), \text { if } 0 \leq \phi<\frac{c}{\alpha \gamma V} \\
1, \text { if } \frac{c}{\alpha \gamma V} \leq \phi \leq 1
\end{array}\right.
$$


It follows that aggregate infection is

$$
I^{S N A}=\left\{\begin{array}{l}
\frac{\alpha}{2}\left(\phi+\frac{c}{\alpha \gamma V}\right), \text { if } 0 \leq \phi<\frac{c}{\alpha \gamma V} \\
\alpha \phi, \text { if } \frac{c}{\alpha \gamma V} \leq \phi \leq 1
\end{array}\right.
$$

It is easy to see that $I^{S N A}=I^{N}$ when $\phi=0$.

\section{Results and Discussion}

The parameters of the model are calibrated to reflect the response to COVID-19 in the United States in the spring/summer of 2020. The calibration assumptions are derived from Acemoglu et al. (2020).

Figures 1 and 2 compare social distancing and aggregate infection under the three policy equilibria described above. Figure 1 uses Equations (11), (15) and (23) to plot the level of social distancing chosen by participating states (nonparticipants do not social distance) as a function of the fraction of states that do not participate. Figure 2 similarly uses Equations (12), (16) and (24) to plot aggregate infection in place of social distancing.

With full participation, $\phi=0$, social distancing in the sub-national agreement is the same as under national policy and is twice as high as under laissez faire. As more states opt out (higher $\phi$ ) the optimal social distancing in participating states increases under both laissez faire and the sub-national agreement; a higher fraction of non-participants implies higher aggregate infection, which induces greater social distancing by participating states. With positive $\phi$, social distancing in the sub-national agreement is always higher than what it would be under the optimal national policy, even though aggregate infection with a national agreement is lower. This happens because the participating states do more to partially make up for the lack of social distancing in non-participating states. Despite more social distancing by the participating states, aggregate infection is still higher. In addition, social distancing under the sub-national agreement is higher than under laissez faire, provided the fraction of non-participants is not too high.

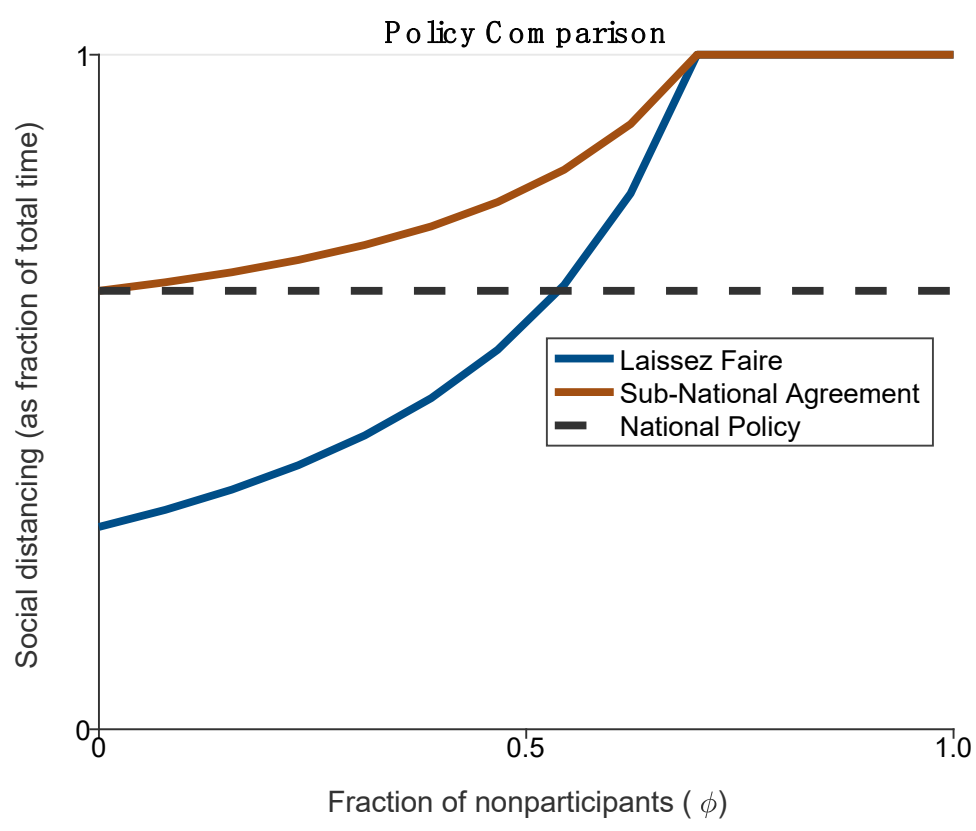

Figure 1. The figure compares policy (for all states) under national policy with policy for participating states in the state-led Laissez Faire regime and in the sub-national agreement. The horizontal axis indicates the fraction of states that do not social distance. 


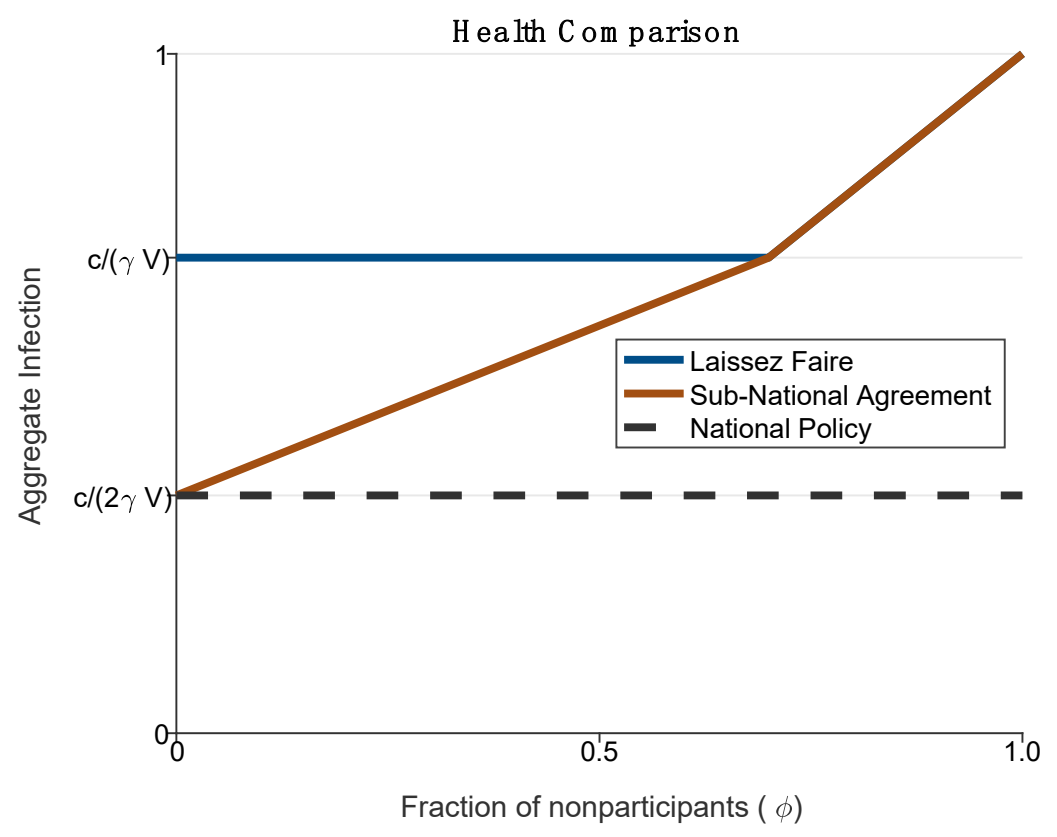

Figure 2. The figure compares aggregate infection under national policy with aggregate infection in the state-led Laissez Faire regime and in a sub-national agreement among participating states. The horizontal axis indicates the fraction of states that do not social distance.

The mandatory national policy always produces the best health outcome, in terms of the lowest aggregate infection, compared to leaving sub-national governments to set social distancing policy (see Figure 2). This is certainly the case if states decide their own policy unilaterally (i.e., the laissez-faire outcome). However, if sub-national governments form a coalition to agree collectively on a social distancing policy, such an agreement can lower substantially aggregate infection as more states participate. If all states join, then their collective action replicates the health outcome of the mandatory national policy. On the other hand, if a large fraction of states do not participate, aggregate infection will rise, and above some threshold of non-participation ( $\phi \geq \frac{c}{\alpha \gamma V}$ in our model), infection will converge to the same level and high rate of increase as under laissez faire.

The results suggest that, absent national leadership, there is a significant role for cooperation among sub-national governments to improve aggregate outcomes. Because controlling disease outbreaks such as COVID-19 through social distancing has strong spillover impacts across jurisdictions within a country, states and provinces that refuse to socially distance impose large costs on other jurisdictions. Moreover, there is a rising economic burden imposed on participating states as the fraction of states that opt out increases. Due to the inter-jurisdictional spillover of the pandemic, sub-national governments that do adopt social distancing have to adopt even more stringent policies, and as a result, face higher costs. Meanwhile, aggregate infection in the country rises as more states and provinces do not impose social distancing. In comparison, if more states and provinces cooperate and adopt a common social distancing policy, not only does the economic burden fall but also aggregate infection declines, eventually approaching the level reached with the optimal national policy.

Our findings do not bode well for countries such as Nigeria, Uruguay, Australia, the United States and Canada, which have displayed great variation in social distancing policies across their states or provinces-suggesting limited cooperation across sub-national jurisdictions (McKenzie and Adams 2020). It may also pose problems for Germany and the United Kingdom, where cooperation among sub-national jurisdictions have shown signs of fragmenting (BBC 2020; Büthe et al. 2020). Other evidence suggests that countries where sub-national governments have undertaken unilateral social distancing measures to combat the pandemic with little or no coordination have performed less well in controlling 
the spread of the disease (Hale et al. 2020b; Imai et al. 2020; GMF Experts 2020; Nouvellet et al. 2020).

Thus, our model strongly supports calls for greater cooperation among and assistance for sub-national governments to improve the effectiveness of their social distancing efforts in controlling the pandemic (GMF Experts 2020; Organization for Economic Cooperation and Development OECD; Pineda and Radics 2020). As Pineda and Radics (2020) conclude, "While autonomous decision-making is a basic feature of decentralization, in areas where the general wellbeing is at stake coordinated action is preferable."

Naturally, the quantitative results in this section are a product of the assumptions made in our relatively simple model. Two assumptions warrant discussion. First, by studying a static model, we abstract from the inherently dynamic nature of the problem under study. A number of recent papers in Economics (see the literature review in the introduction) have studied the problem of social distancing using the workhorse Susceptible-InfectedRecovered (SIR) model from epidemiology. Ideally, we would do the same here, though it would drastically complicate the analysis. While we do not intend to pursue this direction, it is worth noting that the equilibrium policy that would come out of a fully dynamic analysis of the problem considered here would have policy differ both in terms of the intensity of social distancing and the duration and timing at which these policies are kept in place. Our model replaces duration of effort with a single static choice variable with continuous intensity. The qualitative results remain interesting and intuitive, but they lack the quantitative precision that would come out of a fully dynamic analysis. The second assumption of possible concern is the assumption of linear mixing across spatial regions. This assumption is discussed further in the concluding section below.

\section{Conclusions}

During the COVID-19 outbreak, the primary public health policy for controlling the spread of the pandemic has been the adoption of social distancing, such as physical distancing rules, stay-at-home orders, school closures, lockdowns, curfews closing of nonessential businesses, banning of mass gatherings, quarantining and similar regulations to restrict person-to-person contact. Moreover, across many countries, it has been largely sub-national governments, such as states, provinces and municipalities, which have been responsible for instigating and enforcing social distancing (Cheng et al. 2020; GMF Experts 2020; Imai et al. 2020; Nouvellet et al. 2020).

To our knowledge, our paper is the first to analyze systematically the potential effectiveness of the sub-national approach to social distancing in controlling the COVID-19 pandemic. Our simple disease outbreak model demonstrates the public health case for adopting social distancing as the key to controlling the initial outbreak of an epidemic and reducing the number of infections and deaths. However, this outcome presumes that social distancing is a mandatory national policy imposed across all jurisdictions of a country. This is one institutional setting that we explore in our model of sub-national decision making on social distancing. The other two cases are a laissez-faire setting whereby each state determines its own social distancing policy, and a sub-national agreement outcome whereby states form a coalition and collectively choose a social distancing policy that they all pursue. The key policy implication to emerge from this analysis is that, in the absence of national leadership or a mandatory policy on social distancing, greater cooperation among sub-national governments can reduce aggregate infection from a new disease outbreak such as COVID-19 as well as reduce the economic burden borne by sub-national jurisdictions.

This suggests that national governments can best support a de-centralized approach to social distancing and other health policies to control the pandemic by actively encouraging and fostering sub-national governments to coordinate and collectively set their policies. Health outcomes enacted at the state or local level, such as control of the COVID-19 disease outbreak through social distancing, are local public goods with strong inter-jurisdictional spillovers (GMF Experts 2020; Pineda and Radics 2020; Ponce-Rodríguez et al. 2018). Studies show that control of disease and other health outcomes with local public good 
characteristics and inter-jurisdictions spillovers are enhanced by sub-national agreements and coordination over policy (Leland et al. 2020; Ponce-Rodríguez et al. 2018; Spicer et al. 2010). However, sub-national governments may need substantial support from central government to enhance coordination (GMF Experts 2020; Organization for Economic Cooperation and Development OECD), and in the case of developing countries, the support may need to come from the international community to strengthen capacity and provide additional resources to assist sub-national governments (Pineda and Radics 2020).

National governments may also need to provide more direct financial assistance to support social distancing policies adopted by sub-national jurisdictions. In OECD countries, sub-national governments are responsible for 55\% of average country expenditure on public order and safety, and there is evidence that states, provinces and municipalities are suffering severe economic burdens from the coronavirus outbreak as it raises both local health service demand and public order spending due to lockdowns and other social distancing measures (Organization for Economic Cooperation and Development OECD). Financial support from central governments could ease this economic burden of social distancing and health policies imposed by sub-national jurisdictions, which as our model shows is disproportionately born by the jurisdictions that adopt such policies.

Further research in these areas is clearly warranted. First, we need more research on the effectiveness and economic costs of de-centralizing social distancing and other policies to combat pandemics and improve health outcomes to sub-national entities. Second, our model focuses on the potential consequences for policy choices of inter-jurisdictional spillovers with respect to controlling a pandemic, such as COVID-19, within a country. However, clearly any pandemic may also spillover across national borders, for example through international travel and migration. During the pandemic, governments imposed 1123 external border restrictions to limit such spillovers (Cheng et al. 2020). The effectiveness of coordinating such national policies across supra-national groupings, such as the European Union, the North American Free Trade Agreement, the Association of Southeast Asian Nations and the African Union, could be an important extension to our analysis. Finally, although our approach is applied to analyzing sub-national social distancing policies enacted to control COVID-19, it is easily extended to include other de-centralized policies enacted by sub-national governments that have large inter-jurisdictional spillovers. These include important educational, health and environmental policies (Leland et al. 2020; Ponce-Rodríguez et al. 2018; Spicer et al. 2010). For example, one promising extension is to climate change policy, where sub-national governments are increasingly taking an active role around the world, especially where there is a lack of leadership or effective policy at the national level (Somanathan et al. 2014).

The simplified form of the analysis warrants two caveats. First, an important limitation of the current model is the assumption that disease mixing across spatial regions is linear. Since disease contagion has the potential to be a highly-nonlinear phenomenon, it would be interesting to see how the quantitative results change if the aggregate infection equation (Equation (1)) were extended to take a nonlinear form. A potentially tractable formulation would be to use assume that aggregate infection is a CES function of jurisdiction-level policy. Second, the analysis does not consider the economic characteristics of different territories where the containment measures are to be implemented, including sectoral structure, labor linkages, and trade linkages (Acemoglu et al. 2020; Barbieri et al. 2020; Caselli et al. 2020; Gauvin et al. 2020; Burzynski et al. 2020; Favero et al. 2020). Future research may further investigate these aspects.

Author Contributions: Conceptualization, T.I. and E.B.; formal analysis, T.I.; writing, T.I. and E.B. Both authors have read and agreed to the published version of the manuscript.

Funding: This research received no external funding.

Data Availability Statement: There is no data to be shared for the main research.

Conflicts of Interest: The authors declare no conflict of interest. 


\section{Appendix A}

Assume a large fixed population of size $N$, which is initially free from a new contagious virus with a risk of mortality (e.g., COVID-19). At any time $t$, the population includes a subset $S(t)$ that is susceptible to the virus, and a subset that is infected $I(t)$. Each infected individual has a probability $\gamma$ of dying, and so deaths among the population are $D(t)=\gamma I(t)$. The average duration of an infection is $E$ (in units of time).

The basic reproduction rate $R_{0}$ of a disease is the expected number of secondary cases per primary case in a population of individuals susceptible to the new disease (Cobey 2020; van den Driessche 2017). Thus, $R_{0}$ is the initial growth rate of the disease in the human population. For many disease models, an explicit expression for $R_{0}$ can be found in terms of the parameters, which is the also the case for the model developed here. We derive this basic reproduction rate as follows.

The contact process is generally modeled by assuming that each individual has a certain expected number of contacts per unit of time $\mathrm{k}$ with other individuals, which is independent of population size. Then, $\frac{K S}{N}$ of these contacts are with susceptible individuals. If the probability that contact results in transmission is denoted as $p$, then each infected individual passes the virus to $\frac{p \kappa S}{N}$ susceptible individuals per unit of time.

Suppose that initially the entire population is susceptible to the virus and one individual is infected, so that $S(0)=N-1$ and infected $I(0)=1$. As all the contacts of this single individual are with susceptible people, then using as dot over a variable to denote the change in that variable with respect to time, whether there is an initial increase in the number of infected individuals can be denoted as

$$
\left.\dot{I}\right|_{I(0)=1}=\frac{\left(R_{0}-1\right)}{E}, R_{0}=p \kappa \frac{S(0)}{N} E=p \kappa E
$$

where we make use of that fact that, for a large population, $S(0) / N=(N-1) / N \approx 1$. As is clear from (A1), whether the initial infection results in an epidemic depends on the basic rate of reproduction $R_{0}$. It has a threshold value 1 , in that an epidemic will result from the introduction of the infective agent if $R_{0}>1$, as the number of infected individuals initially starts growing exponentially at rate $\left(R_{0}-1\right) / E$. It follows that the expected number of deaths also grow exponentially at the rate $\dot{D}=\gamma \dot{I}$. In comparison, if $R_{0} \leq 1$ then there is no initial spreading of the infection. For many disease models, an explicit expression for $R_{0}$ in (1) can be derived in terms of the parameters of the model (Hethcote 2009; van den Driessche 2017). For example, in the classic susceptible- infected-removed (SIR) model, the disease transmission rate is $\beta=p \kappa / N$, the recovery rate is $v>0$, and the average duration of an infection is $E=1 / v$; consequently, $R_{0}=\beta / v$.

The total number of susceptible individuals who succumb to the virus in the course of the epidemic is also determined by $R_{0}$. This is particularly the case if it is unclear whether recovery from the virus confers immunity to an individual, and so all individuals are either infected or susceptible to infection. In a constant population, and with infection spreading to more and more individuals, the number of people who are susceptible to the virus can only decrease, and so in the long run $S(t)$ must converge to a finite value. It follows that there must also be a finite number of individuals who are infected sooner or later, with some of them expected to die with probability $\gamma$. To show this, we adapt the approach of Diekmann and Heesterbeek (2000).

Defining $s=\frac{S}{N}$, we denote $s^{*}$ as the proportion of the population at the end of the outbreak that is still susceptible to the virus. Consequently, $I^{*}=N\left(1-s^{*}\right)$ is the final size of the population that became infected in the epidemic, and the expected number of deaths are $\gamma I^{*}$. At time $t_{0}$, an individual that is susceptible and experiences a force of infection $\lambda(t)$ for $t>t_{0}$ will escape from being infected with probability

$$
F(t)=e^{-\int_{t_{0}}^{t} \lambda(\tau) d \tau}, \frac{d F}{d t}=-\lambda F, F\left(t_{0}\right)=1
$$


As the population is large, in the long run $s^{*}=\lim _{t \rightarrow \infty} F(t)=F^{*}$, i.e., the fraction that remains susceptible to the virus equals the probability to remain susceptible. It follows that $\int_{t_{0}}^{\infty} \lambda(\tau) d \tau$ can be defined as the total cumulative force of infection. As the susceptible individual has contacts with others with probability $\frac{1}{N}$, the fraction of the population that is infected in the long run $i^{*}$ generates a total cumulative force of infection equal to

$$
\frac{1}{N}(p \kappa E) I^{*}=\frac{R_{0} I^{*}}{N}
$$

Hence

$$
s^{*}=F^{*}=e^{-R_{0}\left(1-s^{*}\right)} \text { and } \ln s^{*}=R_{0}\left(s^{*}-1\right) .
$$

when $R_{0}>1$ and an epidemic occurs, then $0<s^{*}<1$. A certain fraction of the population escapes from ever getting infected with the virus, and that fraction is completely determined by $R_{0}$. The larger $R_{0}$ is, the smaller is the fraction that escapes, and it is negligibly small for large values of $R_{0}$. Consequently, if $R_{0}$ is large, the final size of the population infected during the epidemic $I^{*}=N\left(1-s^{*}\right)$ will be significant and the number of expected deaths $v I^{*}$ will also be high. This appears to be the experience with the COVID-19 outbreak around the world.

As Equations (A1) and (A4) indicate, the key to controlling the initial outbreak of an epidemic and reducing the number of infections and deaths is through reducing $R_{0}$.

Assume that the population $N$ lives in a single jurisdiction $i$, and chooses a level of social distancing $d_{i}$. We choose units of $d_{i}$ so that the contact rate becomes $\kappa\left(1-d_{i}\right)$. The more stringent the social distancing policy chosen, the lower the contact rate between infected and susceptible individuals. Even if social distancing is incapable of stopping the epidemic from occurring, i.e., $R_{0}>1$ still, Equation (A1) indicates that the policy will still slow down the initial spread of the virus as the growth rate declines to $\left(\left(1-d_{i}\right) R_{0}-1\right) / E$. It follows that the initial growth in expected number of deaths is also lower. Similarly, the second equation in (A4) now becomes

$$
\ln s^{*}=\left(1-d_{i}\right) R_{0}\left(s^{*}-1\right)
$$

As $\left(1-d_{i}\right) R_{0}<R_{0}$, a larger fraction of the population $s^{* *}>s^{*}$ escapes from ever getting infected with the virus. Consequently, the final number of infected individuals $I^{* *}=N\left(1-s^{* *}\right)$ is lower, and so are the expected numbers of deaths $v I^{* *}$.

\section{References}

Acemoglu, Daron, Victor Chernozhukov, Iván Werning, and Michael Whinston. 2020. A Multi-Risk SIR Model with Optimally Targeted Lockdown. NBER Working Paper w27102. Cambridge: National Bureau of Economic Research.

Adolph, Christopher, Kenya Amano, Bree Bang-Jansen, Nancy Fullman, and John Wilkerson. 2020. Pandemic Politics: Timing State-level Social Distancing Responses to COVID-19. medRxiv, March 31. [CrossRef]

Atkeson, Andrew. 2020. What will Be the Economic Impact of COVID-19 in the US? Rough Estimates of Disease Scenarios. No. w26867. Cambridge: National Bureau of Economic Research.

Ball, Frank, Denis Mollison, Gianpaolo Scalla-Tomba, Mark Newman, Albert-László Barabási, and Duncan J. Watts. 2011. Epidemics with two levels of mixing. In The Structure and Dynamics of Networks. Princeton: Princeton University Press, pp. 436-79.

Barbieri, Teresa, Gaetano Basso, and Sergio Scicchitano. 2020. Italian Workers at Risk During the Covid-19 Epidemic. GLO Discussion Paper Series 513; Essen: Global Labor Organization (GLO).

BBC. 2020. Coronavirus: How Scotland's Lockdown Rules differ from the other Nations. BBC Neww. May 19. Available online: https:/ / www.bbc.com/news/uk-scotland-52616182 (accessed on 4 June 2020).

Burzynski, Michal, Joel Machado, Atte Aalto, Michel A. R. Beine, Jorge Goncalves, Tom Haas, Francoise Kemp, Stefano Magni, Laurent Mombaerts, Pierre M. Picard, and et al. 2020. COVID-19 Crisis Management in Luxembourg: Insights from an Epidemionomic Approach. LISER Working Paper Series 2020-08; Luxembourg: LISER.

Büthe, Tim, Luca Messerschmidt, and Cindy Cheng. 2020. Policy responses to the coronavirus in Germany. In The World Before and After COVID-19: Intellectual Reflections on Politics, Diplomacy and International Relations. Edited by Gian Luca Gardini. Stockholm and Salamanca: European Institute of International Studies/Instituto Europeo de Estudios Internacionales.

Caselli, Mauro, Andrea Fracasso, and Sergio Scicchitano. 2020. From the Lockdown to the New Normal: An Analysis of the Limitations to Individual Mobility in Italy Following the Covid-19 Crisis. GLO Discussion Paper Series 683; Essen: Global Labor Organization (GLO). 
Cheng, Cindy, Joan Barceló, Allison Hartnett, Robert Kubinec, and Luca Messerschmidt. 2020. COVID-19 Government Response Event Dataset. Nature Human Behavior 4: 756-68.

Chu, Derek, Stephanie Duda, Karla Solo, Sally Yaacoub, and Holger J. Schünemann. 2020. Physical distancing, face masks, and eye protection to prevent person-to-person transmission of SARS-CoV-2 and COVID-19: A systematic review and meta-analysis. The Lancet 395: 1973-87. [CrossRef]

Cobey, Sarah. 2020. Modeling infectious disease dynamics. Science 368: 713-714. [CrossRef]

Copeland, Brian, and Scott Taylor. 1995. Trade and transboundary pollution. The American Economic Review, 716-737.

Diekmann, Odo, and Johan Andre Peter Heesterbeek. 2000. Mathematical Epidemiology of Infectious Diseases: Model Building, Analysis and Interpretation. Chichester: John Wiley.

Eichenbaum, Martin, Sergio Rebelo, and Mathias Trabandt. 2020. The Macroeconomics of Epidemics. No. w26882. Cambridge: National Bureau of Economic Research.

Eubank, Stephan, Spencer Lewis, Marathe Vankatramanan, and Christopher Barrett. 2020. Commentary on Ferguson, et al., "Impact of Non-pharmaceutical Interventions (NPIs) to Reduce COVID-19 Mortality and Healthcare Demand". Bulletin of Mathematical Biology 82: 52. [CrossRef] [PubMed]

Favero, Carlo A., Andrea Ichino, and Aldo Rustichini. 2020. Restarting the Economy While Saving Lives Under Covid-19 (April 2020). CEPR Discussion Paper No. DP14664. Available online: https://ssrn.com/abstract=3594296 (accessed on 8 June 2020).

Ferguson, Neil M., Daniel Laydon, Gemma Nedjati-Gilani, Natsuko Imai, Kylie Ainslie, M. Baguelin, and C. Ghani Azra. 2020. Report 9: Impact of non-pharmaceutical interventions (NPIs) to reduce COVID-19 mortality and healthcare demand. Imperial College London, March 16. [CrossRef]

Gauvin, Laetitia, Paolo Bajardi, Emanuele Pepe, Brennan Lake, Filippo Privitera, and Michele Tizzoni. 2020. Socioeconomic determinants of mobility responses during the first wave of COVID-19 in Italy: From provinces to neighbourhoods. medRxiv, November 18 .

GMF Experts. 2020. How well Do National and Sub-National Governments Cooperate on Coronavirus? German Marshall Fund of the United States. 3 April 2020. Available online: https:/ /www.gmfus.org/blog/2020/04/03/transatlantic-take-360-how-well-donational-and-sub-national-governments-cooperate (accessed on 18 July 2020).

Hale, Thomas, Noam Angrist, Beatriz Kira, Anna Petherick, Toby Phillips, and Samuel Webster. 2020a. Variation in Government Responses to COVID-19, Version 6.0. Blavatnik School of Government Working Paper. Oxford: Blavatnik School of Government, University of Oxford, Available online: www.bsg.ox.ac.uk/covidtracker (accessed on 10 July 2020).

Hale, Thomas, Toby Phillips, Anna Petherick, Beatriz Kira, Noam Angrist, Katy Aymar, and Samuel Webster. 2020b. Lockdown Rollback Checklist: Do Countries Meet WHO Recommendations for Rolling Back Lockdown? Version 4.0 Blavatnik School of Government Working Paper. Oxford: Blavatnik School of Government, University of Oxford.

Hethcote, Herbert W. 2009. The basic epidemiology models: Models, expression for $\mathrm{R}_{0}$, parameter estimation, and applications. Mathematical Understanding of Infectious Disease Dynamics 16: 1-61.

Holtz, David, Michael Zhao, Seth G. Benzell, Cathy Y. Cao, Mohammad Amin Rahimian, Jeremy Yang, Jennifer Allen, Avinash Collis, Alex Moehring, Tara Sowrirajan, and et al. 2020. Interdependence and the cost of uncoordinated responses to COVID-19. Proceedings of the National Academy of Sciences 117: 19837-43. [CrossRef] [PubMed]

Imai, Natsuko, Katy A.M. Gaythorpe, Sam Abbott, Sangeeta Bhatia, Sabine van Esland, Kiesha Prem, Yang Liu, and Neil M. Ferguson. 2020. Adoption and impact of non-pharmaceutical interventions for COVID-19. Wellcome Open Research 5: 59. [CrossRef] [PubMed]

Leland, Suzanne, Jacqueline Chattopadhyay, Cherie Maestas, and Jaclyn Piatak. 2020. Policy venue preference and relative trust in government in federal systems. Governance, 1-21. [CrossRef]

Lin, Zhixian, and Christopher Meissner. 2020. Health vs. Wealth? Public Health Policies and the Economy During Covid-19. No. w27099. Cambridge: National Bureau of Economic Research.

McKenzie, Grant, and Benjamin Adams. 2020. A country comparison of place-based activity response to COVID-19 policies. arXiv arXiv:2005.08738. [CrossRef] [PubMed]

Nouvellet, Pierre, Sangeeta Bhatia, Anne Cori, Kylie E. C. Ainslie, and Marc Baguelin. 2020. Report 26: Reduction in mobility and COVID-19 transmission. Imperial College London, June 8. [CrossRef]

Organization for Economic Cooperation and Development (OECD). 2020a. COVID-19 and Fiscal Relations Across Levels of Government. Paris: OECD, Available online: https: / / read.oecd-ilibrary.org/view /?ref=129_129940-barx72laqm\&title=COVID-19-and-FiscalRelations-across-Levels-of-Government (accessed on 5 July 2020).

Organization for Economic Cooperation and Development (OECD). 2020b. The Territorial Impact of COVID-19: Managing the Crisis Across Levels of Government. Paris: OECD, Available online: https: / /read.oecd-ilibrary.org/view / ?ref=128_128287-5agkkojaaa\& title=The-territorial-impact-of-covid-19-managing-the-crisis-across-levels-of-government (accessed on 5 November 2020).

Peri, Alessandro, Terrence Iverson, and Larry Karp. 2021. Optimal Social Distancing and the Economics of Uncertain Vaccine Arrival. SSRN Working Paper \#3754054. Amsterdam: Elsevier.

Pineda, Emilio, and Axel Radics. 2020. Subnational Governments and Coronavirus: Five Critical Actions the IDB is Supporting. Recaudando Bienestar, Inter-American Development Bank 29 April 2020. Available online: https://blogs.iadb.org/gestion-fiscal/ en/subnational-governments-and-coronavirus-five-actions-idb-is-supporting/\#_ftn4 (accessed on 3 December 2020). 
Ponce-Rodríguez, Raúl A., Charles R. Hankla, Jorge Martinez-Vazquez, and Eunice Heredia-Ortiz. 2018. Rethinking the political economy of decentralization: How elections and parties shape the provision of local public goods. Publius: The Journal of Federalism 48: 523-58. [CrossRef]

Sandler, Todd. 1997. Global Challenges: An Approach to Environmental, Political, and Economic Problems. Cambridge: Cambridge University Press.

Somanathan, Eswaran, Thomas Sterner, and Taishi Sugiyama. 2014. National and sub-national policies and institutions. In Climate Change 2014: Mitigation of Climate Change. Contribution of Working Group III to the Fifth Assessment Report of the Intergovernmental Panel on Climate Change. Edited by O. Edenhofer. Cambridge and New York: Cambridge University Press.

Spicer, Neil, Julia Aleshkina, Regien Biesma, Ruairi Brugha, Carlos Caceres, Baltazar Chilundo, Ketevan Chkhatarashvili, Andrew Harmer, Pierre Miege, Gulgun Murzalieva, and et al. 2010. National and subnational HIV / AIDS coordination: Are global health initiatives closing the gap between intent and practice? Globalization and Health 6: 3. [CrossRef] [PubMed]

van den Driessche, Pauline. 2017. Reproduction numbers of infectious disease models. Infectious Disease Modelling 2: $288-303$. [CrossRef] [PubMed] 